\title{
A CLASSIFICAÇÃO BRASILEIRA DE OCUPAÇÕES DO PROFISSIO- NAL DE EDUCAÇÃO FÍSICA NO SUS: DA INCOMPATIBILIDADE À PROVISORIEDADE NOS SERVIÇOS PÚBLICOS DE SAÚDE
}

\author{
Alessandra Xavier Bueno \\ Universidade Federal do Rio Grande do Sul, Porto Alegre, Rio Grande do Sul, Brasil \\ Cibele Biehl Bossle \\ Universidade Federal do Rio Grande do Sul, Porto Alegre, Rio Grande do Sul, Brasil \\ Alex Branco Fraga \\ Universidade Federal do Rio Grande do Sul, Porto Alegre, Rio Grande do Sul, Brasil
}

\begin{abstract}
Resumo
Este artigo trata de analisar a incompatibilidade dos códigos da Classificação Brasileira de Ocupações (CBO) da Educação Física na saúde inseridos em 2008 nas portarias de implantação dos Núcleos de Apoio à Saúde da Família (NASF), bem como a criação, por parte do Ministério da Saúde (MS), do código da CBO provisório em 2013. Consiste em pesquisa qualitativa realizada através de análise de documentos do Ministério do Trabalho, MS e Conselho Federal de Educação Física. O estudo identificou uma desconexão entre a nomenclatura das ocupações atribuídas à Educação Física, a limitação da atuação no serviço de saúde em pauta e o impacto positivo do código provisório publicado pelo Ministério da Saúde em 2013.
\end{abstract}

Palavras-chave: Educação Física e treinamento. Sistema Único de Saúde. Análise qualitativa. Sistemas de informação em saúde. Ocupações em saúde.

\section{Introdução}

Em 2013, o Ministério da Saúde (MS) instituiu um código provisório da Classificação Brasileira de Ocupações (CBO) para o cadastro dos profissionais de Educação Física nos serviços de saúde, através da Portaria GM no 256, de 11 de março, intitulado 'Profissional de Educação Física na Saúde’ (2241-E1) (BRASIL, 2013a). Nesta mesma portaria, em parágrafo único referente ao código provisório, a Secretaria de Atenção à Saúde (SAS) reconhece tanto a licenciatura quanto o bacharelado em Educação Física como habilitações válidas para a atuação deste profissional no campo da saúde. A intenção do MS, à época, era eliminar a incongruência entre as atividades requeridas ao profissional desta área, especialmente na Atenção Básica, e o que ele podia registrar nos sistemas de informação do Sistema Único de Saúde (SUS). Este artigo trata, portanto, de analisar a incompatibilidade dos códigos da CBO da Educação Física na saúde inseridos em 2008 nas portarias de implantação dos Núcleos de Apoio à Saúde da Família (NASF), bem como a criação, por parte do MS, do código da CBO provisório em 2013.

A CBO é o "documento normalizador do reconhecimento (no sentido classificatório), da nomeação e da codificação dos títulos e conteúdos das ocupações do mercado de trabalho 
brasileiro". A CBO não tem função de regulação profissional, é uma classificação enumerativa e descritiva ao mesmo tempo. É referência obrigatória dos registros administrativos que vão alimentar os diversos programas da política de trabalho do país. É uma ferramenta relevante para a organização dos dados estatísticos que vão desde emprego-desemprego, taxas de natalidade-mortalidade das ocupações, elaboração de currículos, planejamento da educação profissional, rastreamento de vagas e serviços de intermediação de mão de obra (BRASIL, 2010a). A responsabilidade de elaboração e atualização da CBO é do Ministério do Trabalho e Emprego (MTE). O Ministério da Saúde adota a CBO como referência para os códigos profissionais de cada área no âmbito do Sistema Único de Saúde (SUS).

$\mathrm{Na} \mathrm{CBO}$ estão listadas as mais variadas ocupações, desde as mais corriqueiras, como açougueiro(a), cabeleireiro(a) e recepcionista, até as mais peculiares, como salgador de alimentos, enfermeiro e confeccionador(a) de piano. Cada ocupação recebe um código correspondente. Nozoe, Bianchi e Rondet (2003, p. 235) explicam que o código ocupacional "é a chave de identificação do emprego, juntamente com a Classificação Nacional de Atividade Econômica - CNAE e da natureza jurídica dos estabelecimentos". Os trabalhadores do SUS são cadastrados nos sistemas de informação logo que iniciam suas atividades e são identificados profissionalmente pelo código da CBO.

O código da CBO é o código de atividade profissional sob o qual cada trabalhador de saúde que atua no SUS é credenciado pelo município no Sistema de Cadastro Nacional de Estabelecimentos de Saúde (SCNES). Está relacionado a uma série de procedimentos previamente estabelecidos pelo MS, possíveis de registro pelos profissionais de cada área. Por exemplo, não é possível para o(a) enfermeiro(a) registrar o procedimento 'Parto Normal em Gestação de Alto Risco' (cód. de procedimento 03.10.01.004-7) no sistema de informações, já que este procedimento não está vinculado ao código da $\mathrm{CBO}$ deste profissional e, sim, a cinco códigos da CBO das especialidades médicas.

No caso da Educação Física, em que pese seu reconhecimento formal pelo Conselho Nacional da Saúde como profissão deste campo, as delimitações de fronteira entre o que se pode ou não fazer, e registrar, nos serviços ainda não são tão precisas quanto na Enfermagem. De acordo com Loch e Florindo (2012), nos últimos anos, houve um considerável aumento nas possibilidades de inserção do profissional de EF na saúde pública. Este aumento pode ser visualizado através dos registros disponíveis no CNES. Por exemplo, em dezembro de 2007 (competência 12/2007), havia cerca de 86 professores/profissionais de EF atuando em estabelecimentos de saúde no Brasil. Já em dezembro de 2015 (competência 12/2015), os registros da CNES contabilizaram 6.215 professores/profissionais de EF. Especificamente em relação ao CBO provisório da EF citado no início deste texto, intitulado 'Profissional de Educação Física na Saúde' (código 2241-E1), observa-se que houve uma imediata substituição de códigos anteriores da EF a partir de março de 2013 - período em que foi publicada a portaria que instituiu o referido código provisório. Neste período, constavam 849 registros e, em dezembro de 2015, passaram a ser computados 4.727 registros do código provisório. Ou seja, dos 6.215 registros de professores/profissionais de EF na saúde, 76,05\% estavam registrados sob o código provisório. Isso demonstra o impacto da Portaria GM n ${ }^{\circ}$ 256/2013 (BRASIL, 2013a) na adequação dos códigos de CBO da EF para o campo da saúde.

Esse código provisório de CBO foi introduzido no sistema de informações do SUS para tentar resolver dificuldades enfrentadas pela gestão dos municípios e pelos profissionais de EF quando do registro dos profissionais e os respectivos procedimentos. Para ter uma ideia destas dificuldades, é preciso compreender como esses registros em relação ao código de CBO vinham sendo feitos antes da instituição da Portaria GM n ${ }^{\circ}$ 256/2013 (BRASIL, 2013a) e, também, o processo que levou o MS a instituir um código da CBO provisório para a EF. 


\section{A CBO e as ocupações da Educação Física nos serviços de saúde}

A estrutura básica da CBO foi elaborada em 1977 como resultado do convênio firmado entre o Brasil e a Organização das Nações Unidas (ONU), por intermédio da Organização Internacional do Trabalho (OIT), no Projeto de Planejamento de Recursos Humanos (Projeto BRA/70/550), tendo como base a Classificação Internacional Uniforme de Ocupações (CIUO) de 1968 (BRASIL, 2010a). Com a alteração dos critérios de agregação das ocupações pela Classificação Internacional (CIUO) de 1988, e um descompasso entre a nomenclatura da CBO da época e dos dados censitários do Instituto Brasileiro de Geografia e Estatística (IBGE) - que utilizava nomenclatura própria -, houve um esforço de articulação entre os órgãos brasileiros na tentativa de unificá-las para que se facilitasse tanto a comparação de dados no território nacional como a comparação das estatísticas geradas em outros países (BRASIL, 2010a).

A partir das iniciativas de unificar as classificações em território nacional, foi instituída em 1994 a Comissão Nacional das Classificações (CONCLA), que constituiu um trabalho conjunto do MTE e do IBGE para elaborar a classificação única. Como resultado deste trabalho, foi publicada a Portaria do MTE ${ }^{\circ}$ 397, de 9 de outubro de 2002, que aprovou a CBO, versão 2002 (BRASIL, 2010a).

Nesta nova versão da CBO, a EF foi contemplada com sete ocupações para o grupo 'Profissionais da Educação Física' (chamado de 'família' pela CBO). A saber: avaliador físico (2241-05), ludomotricista (2241-10), preparador de atleta (2241-15), preparador físico (2241-20), técnico de desporto individual e coletivo, exceto futebol (2241-25), técnico de laboratório e fiscalização desportiva (2241-30), treinador profissional de futebol (2241-35). Essas ocupações receberam contribuições do Conselho Federal de Educação Física (CONFEF, 2002b) que, em sua visão, seriam mais condizentes à nova configuração profissional:

No ano de 2000, identificamos que a Classificação Brasileira de Ocupações - CBO estava em processo de modificação, tendo em vista o estabelecimento de nova classificação, imediatamente, nos debruçamos sobre a matéria divulgando e informando à categoria profissional como seria esse processo e de que forma se poderia estar intervindo junto ao Ministério do Trabalho, visto que a Profissão de Educação Física, no Brasil, está mudando no sentido de acompanhar as transformações que se processam em nossa sociedade (CONFEF, 2002b).

Até o ano de 2002, o MTE previa para a EF somente códigos da CBO relativos aos magistérios fundamental, médio e superior: professor de Educação Física no Ensino Fundamental (2313-15); professor de Educação Física no Ensino Médio (2321-20) e professor de Educação Física no Ensino Superior (2344-10). Portanto, toda e qualquer inserção de profissionais da EF no SUS pelos municípios brasileiros, e consequentemente o registro das respectivas atividades realizadas, era feita por meio destes códigos de CBO (docência). Com a reformulação de 2002 da CBO pelo MTE, o MS não retirou dos seus sistemas de informação os códigos relativos à docência, apenas acrescentou as 'novas' designações de ocupação da EF. Dessa forma, passava a ser possível o cadastramento de profissionais ou professores de Educação Física nos serviços sob qualquer um dos códigos de CBO disponíveis no sistema, cabendo ao gestor local (município) a escolha do código mais apropriado para o registro. Com a criação dos NASF em 2008 (BRASIL, 2008a) como um novo serviço da Atenção Básica, foram 'enxertados' nos documentos de regulamentação os códigos das sete ocupações para o EF oriundos da atualização da CBO de 2002 (BRASIL, 2008c). A publicação por portaria destas ocupações, excluindo da listagem os códigos de professor, forçou os gestores locais a cadastrarem os profissionais da EF nos sistemas de informação em um dos sete códigos de 
CBO da EF previstos, mesmo que a gestão dos serviços de Atenção Básica entendesse que a nomenclatura mais condizente com a atividade desempenhada fosse a que vinha sendo usada para o professor.

Os NASF consistem em equipes multiprofissionais que atuam de forma integrada com as Equipes da Estratégia de Saúde da Família (ESF), com equipes da Atenção Básica para populações específicas (BRASIL 2008a), como, por exemplo, consultório de rua, equipes ribeirinhas e fluviais, bem como com o Programa Academia da Saúde (BRASIL, 2013b). A ESF tem como principal propósito "reorganizar a prática da atenção à saúde em novas bases e substituir o modelo tradicional, levando a saúde para mais perto das famílias e, com isso, melhorar a qualidade de vida da população" (BRASIL, 2010b). A atuação nos NASF se dá em equipes multiprofissionais que são compostas por pessoas que têm formação na área da saúde, tais como fisioterapeutas, assistentes sociais, nutricionistas, terapeutas ocupacionais, farmacêuticos, fonoaudiólogos, médicos, psicólogos, profissionais de Educação Física, sanitaristas, dentre outros. É um serviço composto por equipe multiprofissional que tem um papel importante no apoio à equipe da ESF nos processos que envolvem o planejamento e a execução das ações de promoção e educação em saúde.

Na Atenção Básica, o NASF foi o primeiro serviço que apontou de modo mais direto a possibilidade de contratação do profissional de EF no âmbito da Atenção Básica. A característica do serviço, descrita principalmente na publicação dos Cadernos de Atenção Básica $\mathrm{n}^{\circ} 27$ (BRASIL, 2010b), prevê um profissional capaz de lidar com o cotidiano do trabalho para além do fazer representado por uma única especialidade da profissão, como, por exemplo, técnico de futebol.

O NASF foi instituído pela Portaria $\mathrm{n}^{\mathrm{o}}$ 154, de 24 de janeiro de 2008. Em 18 de março do mesmo ano, a portaria foi republicada com uma substancial alteração em relação à EF; foi retirada do texto a designação de 'professor' e foi inserida no lugar a de 'profissional'.

A republicação da portaria com tal alteração coincide com as demandas do sistema $\mathrm{CREF} / \mathrm{CONFEF}$ em relação à diferenciação entre as habilitações de licenciatura e bacharelado, cabendo ao professor de EF a atuação nas escolas e ao profissional os 'demais' campos de atuação fora dela. Não foram encontrados documentos que justificassem a alteração à época, mas não demorou muito para se perceber seus efeitos na organização dos serviços de saúde.

O impacto para a atuação da EF nos serviços de saúde, após a publicação da lista dos sete códigos de $\mathrm{CBO}$ restritos aos profissionais de $\mathrm{EF}$, naquele momento representava uma perda maior para os serviços de saúde, já que a tabela de procedimentos possíveis para registro, paradoxalmente, reduziu-se. Dos 18 procedimentos até então vinculados ao código do professor de EF no Ensino Superior, por exemplo, sobraram apenas três. Ou seja, antes, o 'professor' de EF podia registrar formalmente no sistema 18 atividades e, com a publicação da portaria (BRASIL, 2008c), as opções para o 'profissional' passaram a estar disponíveis em apenas três atividades, dependendo da ocupação cadastrada pelo município referente a este profissional ${ }^{1}$.

Cada uma das sete ocupações do profissional de EF era identificada por um código da $\mathrm{CBO}$, e era permitida a inclusão de apenas um código para cada profissional de EF no NASF que, em teoria, condizia com a atuação deste profissional no serviço de saúde. Ou seja, quando um NASF era habilitado em um município, era possível cadastrar junto ao SCNES apenas uma ocupação para cada profissional de EF. Não havia um código da CBO genérico para cadastro, e o município devia fazer uma escolha entre as sete ocupações descritas na Portaria $\mathrm{n}^{\circ}$ 409/2008. Esse fato gerava um descompasso entre a nomenclatura da 'especificidade' da EF e as atividades e arranjos de trabalho sugeridos pelas diretrizes do NASF.

\footnotetext{
${ }^{1}$ As questões referentes aos procedimentos possíveis de registro para a Educação Física no SUS foram abordadas de modo mais detalhado em BUENO (2012, 2016).
} 


\section{Aspectos metodológicos}

A análise de conteúdo de caráter qualitativo foi a metodologia utilizada na pesquisa que deu origem ao presente artigo. Para Bardin (2004), este tipo de análise pode ser entendido como um conjunto de técnicas de análise das comunicações, que usa procedimentos objetivos e sistemáticos para descrever o conteúdo das mensagens, visando à obtenção de conhecimentos relativos às suas condições de produção e recepção.

O corpo de análise foi composto por documentos do Ministério do Trabalho e Emprego (MTE) relativos à CBO e do Ministério da Saúde relativos ao NASF. Em relação aos materiais do NASF, nosso locus de investigação foi a Portaria $\mathrm{n}^{\circ}$ 154, de 24 de janeiro de 2008 (BRASIL, 2008a), que cria os NASF, e a Portaria $n^{\circ}$ 409/SAS/MS, de 23 de julho de 2008 (BRASIL, 2008c), que indica os códigos de CBO para serem utilizados por diferentes profissões, inclusive a EF. Também foram analisadas as resoluções do CONFEF de 1999 a 2011 e a publicação da mesma instituição intitulada "Recomendações sobre condutas e procedimentos do profissional de educação física na Atenção Básica em saúde", de 2010. Além disso, utilizamos como fonte de dados o instrutivo dos NASF publicado em 2010 (BRASIL, 2010b), material relevante para a implementação e o entendimento do serviço em questão. Também analisamos as relações de códigos da $\mathrm{CBO}$ e os procedimentos possíveis de registro, a partir da versão para download do Sistema de Gerenciamento da Tabela de Procedimentos, Medicamentos e OPM do SUS (SIGTAP). A partir da leitura desses materiais, selecionamos os trechos mais significativos e partimos para a análise propriamente dita.

\section{Análise e discussão}

É possível afirmar que a incompatibilidade dos códigos da CBO do profissional de EF disponível para o registro das atividades destes profissionais interferiu nas práticas de cuidado em serviço e no conhecimento que vinha sendo produzido na área da saúde, não fosse assim o MS não teria se empenhado em elaborar um CBO provisório em 2013 e o mantido ativo por tanto tempo. Dois tipos de interferência, entre tantos, podem ser aqui destacados: o primeiro seria o direcionamento da atuação do profissional de Educação Física, pois, com a diminuição do escopo de atividades (passaram a ser apenas três) vinculadas aos códigos da CBO, a tendência seria centrar o fazer 'naquilo que é possível registrar'. O segundo seria a desistência da contratação de um profissional de Educação Física, pois, na medida em que só é possível registrar três atividades, seria bem conveniente para o gestor do serviço usar o mesmo recurso para pagar um profissional mais versátil, que pudesse fazer (e consequentemente registrar) mais do que três procedimentos. Para Nozoe, Bianchi e Rondet (2003, p.245), "a elaboração de políticas públicas consistentes com a nossa realidade socioeconômica depende da qualidade da informação que recebem seus formuladores". Ao analisar a Portaria no 409/2008, também foi possível perceber que algumas das ocupações descritas para o profissional de EF pareciam estar deslocadas do contexto da Atenção Básica (o nível de atenção nos quais estão inseridos a ESF e, consequentemente, o NASF). A ocupação 'Treinador de Futebol', por exemplo, parecia demasiadamente específica para estar na Atenção Básica. Este código de CBO foi criado para atender a uma demanda do campo de atuação profissional ligado ao futebol, que no Brasil é praticamente um mundo à parte dentro do esporte, mas cujo escopo não guardava relação direta com a atenção à saúde. Além disso, a pura e simples adoção da lista de códigos de $\mathrm{CBO}$ da EF poderia abrir uma brecha para a atuação dos portadores do registro de provisionado ${ }^{2}$ no SUS, caso, por exemplo, um município lançasse um edital de concurso

\footnotetext{
${ }^{2} \mathrm{O}$ registro de provisionado em EF foi instituído para permitir que uma pessoa sem diploma, mas com experiên-
} 
para o cargo de profissional de Educação Física para o NASF sem indicar a necessidade de graduação em Educação Física, constando apenas a exigência do registro no sistema CREF/CONFEF. Apesar de pouco provável, seria possível um técnico de futebol com registro de provisionado ser contratado como trabalhador do NASF, mas não para um licenciado em EF. Em função deste tipo de contradição, o MS se viu obrigado a publicar a Portaria GM n ${ }^{\circ}$ 256, de 11 de março de 2013, instituindo o código provisório da CBO 2241-E1, na qual fica explícita a necessidade de diploma de graduação para este profissional, podendo tanto ser a habilitação licenciatura quanto bacharelado, o que motivou o sistema CREF/CONFEF a levantar uma série de questionamentos sobre a validade desta medida, especialmente no que se refere à atuação do licenciado no SUS. Este é um tema interessantíssimo para o desenvolvimento de futuras pesquisas no campo da $\mathrm{EF}$, especialmente se levarmos em consideração a existência da figura do provisionado, algo que não foi possível discutir neste texto em função do foco do artigo.

Quando se comparam as nomenclaturas propostas pelo CONFEF para identificação das especificidades dentro da profissão de EF com a lista do $\mathrm{CBO}$ da área implantada pelo MTE no mesmo ano, percebe-se que existem muitas similaridades. Por exemplo, para a especificidade 'preparação física' temos um código da CBO de preparador físico, para 'avaliação física', um código de avaliador físico, para a especificidade 'recreação em atividade física', o código de ludomotricista, entre outros. A Resolução no 046 lança sete especificidades e a CBO traz sete ocupações para o profissional de EF. Nenhuma relativa ao trabalho em saúde.

Parece relevante problematizar o que estas nomenclaturas de 'especificidades' da EF, que na CBO se tornaram ocupações, comunicavam aos gestores, trabalhadores e usuários. É possível visualizar que dificilmente um gestor que precisasse contratar um profissional de EF para compor uma equipe de NASF, trabalhando em conjunto com cinco, oito ou mais equipes da ESF, entendesse que um profissional que trabalha especificamente como treinador de futebol fosse adequado para ocupar a vaga de EF em tal equipe. Para isto, é mais coerente pensar na contratação de um profissional com escopo de atuação ampliado, que pudesse compor (com) a equipe, contribuindo para a construção de práticas de cuidado a partir de uma visão ampla sobre os processos de produção de saúde, procurando estabelecer relações com o conhecimento produzido na área da EF. Tal modo de posicionar cada uma das profissões que compõem os serviços é importante pelo fato de que o cotidiano do trabalho em saúde pode vir a demandar formas de intervenção não previstas quando da contratação de um determinado profissional. Além disso, a escolha de uma ocupação entre aquelas indicadas na CBO pelo MTE restringe a potencialidade do que pode ser feito em nome da EF no SUS, seja no NASF, seja em hospitais ou em qualquer outro serviço de saúde vinculado ao setor público.

A análise das alterações dos códigos de $\mathrm{CBO}$ relativos à $\mathrm{EF}$, disparada especialmente pela publicação dos documentos do NASF, demonstra a relação conflitante com a especificidade da nomenclatura utilizada e as perspectivas de atuação da EF nos NASF. Pasquim (2010, p. 195) já havia afirmado que existia "uma má identificação da educação física na Classificação Brasileira de Ocupações (CBO), para a qual não existe uma orientação geral para cadastro no SUS". A análise realizada nos permitiu visualizar que a nomenclatura dada às sete ocupações do profissional de $\mathrm{EF}$ na $\mathrm{CBO}$, presumivelmente influenciada pelos materiais do $\mathrm{CON}$ FEF, especialmente a Resolução n 046 de 2002, reforça esta noção de má identificação da EF no campo da saúde, já que estas nomenclaturas são incompatíveis com o trabalho desenvolvido pelo profissional da EF no NASF, por exemplo. Apesar da dificuldade em encontrar materiais que relatassem o processo de construção desta lista de CBO, é possível inferir que nestas

cia comprovada, pudesse atuar como profissional da área (CONFEF, 2002a). Esse registro beneficiava somente aqueles que atuavam na profissão antes de sua regulamentação, ocorrida em $1^{\circ}$ de setembro de 1998 , e tem sido utilizado em diversas profissões. 
sete ocupações do profissional de EF não foi levada em consideração a discussão do trabalho em saúde na Atenção Básica no SUS. Quanto ao fato de a Portaria nº . 409/2008 ter inserido as ocupações relativas ao profissional de Educação Física restringido o número de procedimentos possíveis de registro, não mais permitindo o uso dos códigos da $\mathrm{CBO}$ anteriores (relativos à docência em EF), é notória a limitação do exercício profissional do licenciado em EF, algo que gera uma série de problemas em relação ao sistema de informação em saúde, por não ser possível o registro de atividades de caráter educativo, por exemplo, que estão acontecendo no cotidiano do serviço de saúde.

\title{
Considerações finais
}

Este estudo problematizou as mudanças que ocorreram nos códigos de CBO da EF com a publicação da Portaria $n^{\circ} 409$, de 2008, que compõe os documentos de implementação do NASF, e analisou a desconexão entre a nomenclatura dos códigos oriundos de sete ocupações listadas no MTE.

Por conta da identificação desta desconexão com as diretrizes do SUS, o Ministério da Saúde publicou um código da CBO provisório para a EF, através da Portaria GM n ${ }^{\circ}$ 256/2013 (BRASIL, 2013a), sob a nomenclatura de 'Profissional de Educação Física na Saúde'. Este código ainda está sendo utilizado somente no âmbito do Ministério da Saúde e foi criado para resolver um problema que, por enquanto, parece estar restrito à atuação deste profissional no campo da saúde.

Em consulta ao SIGTAP, foi possível verificar a lista de procedimentos para este código da CBO provisório. Ao todo são 20 procedimentos - uma possibilidade maior que o código da CBO do Professor de EF no Ensino Superior, utilizado pelo SUS até 2008, cuja listagem de procedimentos relacionados contava com 18 atividades possíveis de registro. Entendemos que o código provisório da CBO para o cadastro dos profissionais de EF é mais condizente com o trabalho nos serviços de saúde. Este novo código é mais generalista e carrega uma única nomenclatura, além disto, amplia as possibilidades de registro das atividades do profissional de EF no SUS em relação aos códigos anteriores.

Os códigos de CBO instituídos em 2002 e 'capturados' para o uso no SUS em 2008 funcionavam como limitadores da atuação do trabalhador de EF na saúde. É possível pensar que os códigos dificultavam também o reconhecimento das atividades desenvolvidas na equipe e subvertiam a ideia de trabalho interdisciplinar. Entretanto, o novo código ainda tem caráter provisório. É necessária uma mobilização de classe profissional e o entendimento da importância desta identificação com o trabalho no SUS para que este código provisório passe a fazer parte da CBO definitiva, junto ao MTE.

\section{THE BRAZILIAN OCCUPATIONAL CLASSIFICATION SYSTEM OF PHYSICAL EDUCATION ON BRAZILIAN HEALTH SYSTEM: FROM INCOMPATIBILITY TO PROVISIONAL APPLICATION}

\begin{abstract}
This article discusses the incompatibility of codes in the Brazilian Classification of Occupations (CBO) for Physical Education professionals on health field incorporated on documents of Unified Health System's Family Health Centers (NASF) by Brazilian Ministry of Health as well as the establishment of a provisional code of CBO in 2013. It is a qualitative study conducted through analysis of documents of the Ministry of Labor, the Ministry of Health, and the Federal Council of Physical Education. The study found a mismatch between the occupation classification attributed to Physical Education, limited professional work by the afore-
\end{abstract}


mentioned health service, and a positive impact of the provisional code published by the Ministry of Health in 2013.

Keywords: Physical Education and training. Unified Health System. Qualitative analysis. Health information systems. Health occupations.

\section{LA CLASIFICACIÓN BRASILEÑA DE EMPLEOS PROFESIONALES DE EDUCA- CIÓN FÍSICA EN EL SISTEMA ÚNICO DE SALUD DE BRASIL: DE LA INCOM- PATIBILIDAD A LA PROVISORIEDAD EN LOS SERVICIOS PÚBLICOS DE SA- LUD}

\section{Resumen}

Este artículo trata de analizar la incompatibilidad de los códigos de la Clasificación Brasileña de Ocupaciones (CBO) de la Educación Física en el campo de la salud insertados en 2008 en los documentos de implantación de los Núcleos de Apoyo a la Salud de la Familia (NASF), así como la creación por parte del Ministerio de Salud (MS) del código de la CBO provisional en 2013. Consiste en la investigación cualitativa mediante el análisis de los documentos del Ministerio de Trabajo, Ministerio de Salud y el Consejo Profesional de Educación Física. El estudio indentificó una desconexión entre la nomenclatura de las ocupaciones asignadas a la Educación Física, la prescripción de las acciones en el servicio de salud de que se trate y el impacto positivo del código provisional publicado por el ministerio de Salud de Brasil en 2013.

Palabras clave: Educación y Entrenamiento Físico. Sistema Único de Salud. Análisis cualitativo. Sistemas de información en salud. Empleos en salud.

\section{Referências}

BUENO, A. X. Entre o fazer e o registrar da Educação Física no NASF: a relação conflitante entre a Classificação Brasileira de Ocupações e os procedimentos possíveis de registro pelo profissional de Educação Física. 2012. 106 f. Dissertação (Mestrado em Educação Física) - Programa de Pós-Graduação em Ciências do Movimento Humano, ESEF/UFRGS, Porto Alegre, 2012.

A Educação Física na saúde: reflexões acerca do fazer da profissão no SUS. In: WACHS, F. ALMEIDA, U.R.; BRANDÃO, F.F (Org.). Educação Física e saúde coletiva: cenários, experiências e artefatos culturais. Porto Alegre: Rede Unida, 2016. p.145-167.

BARDIN, Laurence. Análise de conteúdo. Lisboa: Edições 70, 2004.

BRASIL. Ministério da Saúde. Portaria n. 154, de 24 de janeiro de 2008. Cria os Núcleos de Apoio à Saúde da Família - NASF. 2008a.

Portaria n. 154 GM, republicada em 4 de março de 2008. 2008b.

Ministério da Saúde. Portaria n. 409/SAS/MS, de 23 de julho de 2008. 2008c.

. Classificação Brasileira de Ocupações: CBO - 2010. 3. ed. Brasília: MTE, SPPE, 2010a. 
Ministério da Saúde. Secretaria de Atenção à Saúde. Departamento de Atenção Básica. Diretrizes do NASF: Núcleos de Apoio à Saúde da Família. Cadernos de Atenção Básica, n.27, Brasília: 2010b.

Ministério da Saúde, Secretaria de Atenção à Saúde. Portaria n. 256, de 11 de março de 2013. Estabelece novas regras para o cadastramento das equipes que farão parte dos Núcleos de Apoio à Saúde da Família (NASF), Sistema de Cadastro Nacional de Estabelecimentos de Saúde (SCNES). 2013a.

Ministério da Saúde. Portaria n. 2.681/GM/MS de 08 de novembro de 2013. Redefine o Programa Academia da Saúde no âmbito do Sistema Único de Saúde (SUS). 2013b.

CONFEF. Conselho Federal de Educação Física. Resolução 045, de 18 de fevereiro de 2002. 2002a.

Resolução 046, de 18 de fevereiro de 2002. 2002 b.

LOCH, M. R.; FLORINDO, A. A. A Educação Física e as residências multiprofissionais em saúde. Editorial. Revista Brasileira de Atividade Física e Saúde, v. 17, n. 2, 2012.

NOZOE, N. H.; BIANCHI, A. M.; RONDET, A. C. A. A nova Classificação Brasileira de Ocupações: anotações de uma pesquisa empírica. São Paulo em Perspectiva, São Paulo, v. 17, n. 3-4, p. 234-246, 2003.

PASQUIM, H. M.. A saúde coletiva nos cursos de graduação em Educação Física. Saúde e Sociedade, São Paulo, v. 19, n. 1, p. 193-200, 2010.

Recebido em: 02/06/2017

Revisado em: 09/09/2017

Aprovado em: 10/09/2017

Endereço para correspondência:

bueno.ax@gmail.com

Alessandra Xavier Bueno

Universidade Federal do Rio Grande do Sul

Av. Paulo Gama, 110

Farroupilha, Porto Alegre - RS

90040-060 